\title{
Can Thermal Nonequilibrium Explain Coronal Loops?
}

\author{
James A. Klimchuk, Judy T. Karpen, and Spiro K. Antiochos \\ NASA Goddard Space Flight Center, Greenbelt, MD 20771
}

Received —

Submitted to the Astrophysical Journal 


\begin{abstract}
Any successful model of coronal loops must explain a number of observed properties. For warm ( $1 \mathrm{MK})$ loops, these include: 1. excess density, 2. flat temperature profile, 3. super-hydrostatic scale height, 4. unstructured intensity profile, and 5. 1000-5000 s lifetime. We examine whether thermal nonequilibrium can reproduce the observations by performing hydrodynamic simulations based on steady coronal heating that decreases exponentially with height. We consider both monolithic and multi-stranded loops. The simulations successfully reproduce certain aspects of the observations, including the excess density, but each of them fails in at least one critical way. Monolithic models have far too much intensity structure, while multi-strand models are either too structured or too long-lived. Our results appear to rule out the widespread existence of heating that is both highly concentrated low in the corona and steady or quasisteady (slowly varying or impulsive with a rapid cadence). Active regions would have a very different appearance if the dominant heating mechanism had these properties. Thermal nonequilibrium may nonetheless play an important role in prominences and catastrophic cooling events (e.g., coronal rain) that occupy a small fraction of the coronal volume. However, apparent inconsistencies between the models and observations of cooling events have yet to be understood.
\end{abstract}

Subject headings: hydrodynamics — Sun: activity — Sun: corona — Sun: UV radiation - Sun: X-rays, gamma rays 


\section{Introduction}

It is well known that much of the plasma in the Sun's corona is confined in distinct loop structures. The arching shape of these loops is defined by the magnetic field, but their thermodynamic properties are determined by the yet-to-be-established mechanism of coronal heating. Our understanding of coronal loops and coronal heating has advanced considerably in recent years, but a number of important questions remain. We report here on an investigation into whether ordinary coronal loops can be explained by a phenomenon known as thermal nonequilibrium. Thermal nonequilibrium occurs whenever steady or quasi-steady heating is highly concentrated at low coronal heights in both legs of a loop. It is believed to play an important role in prominences (e.g., Antiochos \& Klimchuk 1991; Karpen, Antiochos, \& Klimchuk 2006), and it seems reasonable to consider that its occurrence is more widespread. Note that quasi-steady heating is here taken to mean heating that changes slowly compared to a cooling time or that is impulsive but repeats rapidly compared to a cooling time.

Early observations of coronal loops were made primarily in soft X-rays and suggested that the loops are in states of static equilibrium (e.g., Rosner, Tucker, \& Vaiana 1978). This implies that the heating is steady. Soft X-ray emission is mostly produced by hot (> $2 \mathrm{MK}$ ) plasma, but more recent observations made in the extreme ultraviolet (EUV)

have revealed a much different picture at lower temperatures. Most warm $(\sim 1 \mathrm{MK})$ loops are clearly inconsistent with static equilibrium. We are referring explicitly to those warm loops that appear as complete structures within the interiors of active regions. We do not consider partial loops, sometimes called "fan" loops, that are often seen at the perimeters of active regions.

A number of discrepancies with static equilibrium have been identified. Perhaps the most significant concerns the density. Static equilibrium theory predicts a well 
defined relationship among the density, temperature, and length of a loop. Warm loops are observed to have a much higher density than is expected given the observed temperature and length (Aschwanden et al. 1999; Aschwanden, Schrijver, \& Alexander 2001; Winebarger, Warren, \& Mariska 2003; Klimchuk 2006). The density excess is typically a factor of about ten, but factors ranging from near unity to more than a thousand have been measured.

A second discrepancy between observations and theory concerns the variation of temperature along the loop. Observations from broad-band and narrow-band imagers such as the Transition Region and Coronal Explorer (TRACE) can be used to measure temperature with a method known as the filter ratio technique. The ratio of intensities obtained in two filters, or band-passes, is related to a temperature under the assumption that the emitting plasma is isothermal. When measured in this way, warm loops tend to have a temperature profile that is much flatter than expected for static equilibrium (Lenz et al. 1999; Aschwanden et al. 1999; Aschwanden, Schrijver, \& Alexander 2001; although see Reale \& Peres 2000).

A third inconsistency is that the density of warm loops decreases with height much more slowly than expected for a gravitationally stratified plasma at the measured temperature. The scale height is too large by up to a factor of two (Aschwanden. Schrijver, \& Alexander 2001). As a consequence, loops have a more uniform brightness than static equilibrium would predict.

There are two additional properties of observed loops that prove extremely important for constraining the models. Both are consistent with static equilibrium. Most loops do not have small-scale intensity structure. With occasional exception, there are no localized bright spots or abrupt transitions in brightness. This is true for both warm loops (López Fuentes, Démoulin, \& Klimchuk 2008) and hot loops (Kano \& Tsuneta 1996; 
Klimchuk 2000).

Finally, there is the loop lifetime. Warm loops are typically visible for 1000-5000 s (Winebarger, Warren, \& Seaton 2003; Winebarger \& Warren 2005; Ugarte-Urra, Winebarger, \& Warren 2006; Ugarte-Urra, Warren, \& Brooks 2009),

though some can live considerably longer. Hot loops have a much larger range of lifetimes, with many persisting for multiple hours (López Fuentes, Klimchuk, \& Mandrini 2007). In all cases the loop lives longer than the cooling time expected from the measured temperature, density, and loop length.

Explaining all five of these observed properties is very challenging. One model that does so successfully postulates that loops are bundles of unresolved strands that are heated impulsively by storms of nanoflares; see Klimchuk $(2006,2009)$ for a discussion of the basic idea and references to key papers. In this picture, each strand is heated once and allowed to cool, but many different strands are energized over a finite time window, which is the storm duration. Impulsive heating is very appealing both because it is able to explain the observations and because all current theories of heating mechanisms predict that the heating is short lived on individual magnetic strands (Klimchuk 2006). This includes wave heating. A critical aspect of the nanoflare storm idea is that strands do not get reheated. The plasma must be allowed to cool from high temperatures to less than $1 \mathrm{MK}$ in order to explain over-dense warm loops. If nanoflares recur in a given strand with a delay that is significantly shorter than a cooling time, then the conditions are similar to steady heating.

In the work presented here, we assume that the heating is truly steady or that the cadence of impulsive heating is sufficiently rapid that a steady approximation is valid. We further assume that the heating is highly concentrated near both footpoints of the loop. Such conditions are known to produce a state of thermal nonequilibrium (Antiochos \& Klimchuk 1991; Antiochos et al. 1999; Karpen et al. 2001, 2003, 2005; Müller, Hansteen, \& Peter 
2003; Müller, Peter, \& Hansteen 2004; Karpen, Antiochos, \& Klimchuk 2006; Mok et al. 2008). As the name implies, no equilibrium exists. The loop is inherently dynamic and undergoes periodic convulsions as it searches for a nonexistent equilibrium. Cold, dense condensations form, slide down the loop leg, and later reform in a cycle that repeats with periods of several tens of minutes to a few hours. It has been firmly established that rapidly repeating, low-altitude nanoflares also produce a state of thermal nonequilibrium (Testa. Peres, \& Reale 2005; Karpen \& Antiochos 2008; Susino et al. 2010; Antolin \& Shibata 2009).

Our objective here is to determine whether thermal nonequilibrium can reproduce the observations described above, in particular the EUV observations of warm loops: 1. excess density, 2. flat temperature profile, 3. super-hydrostatic scale height, 4. unstructured intensity profile, and 5. 1000-5000 s lifetime. Our approach is to perform numerical loop simulations by solving the 1D time-dependent hydrodynamic equations. From these, we generate synthetic data representing observations made in the 171 and 195 channels of TRACE and the Al.1 and AlMg channels of the Soft X-ray Telescope (SXT) on Yohkoh. We then measure temperature and density using precisely the same filter ratio technique that is applied to real data.

Our study treats two fundamentally different types of loops. We first consider monolithic structures in which the plasma is uniform over the loop cross section. We then consider bundles of very thin unresolved strands, similar to what is envisioned in the nanoflare storm picture described above. Just as the nanoflares are assumed to occur at different times, we assume that the condensation cycles of thermal nonequilibrium are out of phase in the different strands. We describe the details of the numerical model in the next section and present and discuss the simulation results in Sections 3 and 4. 


\section{Numerical Model}

Because the solar corona is highly ionized and because the magnetic field dominates the plasma within active regions (i.e., the plasma $\beta$ is small), we can treat coronal loop strands as one-dimensional structures. We therefore perform our numerical simulations

with the Adaptively Refined Godunov Solver (ARGOS) hydro code (Antiochos et al. 1999), which solves the 1D hydrodynamic equations of mass, momentum, and energy conservation:

$$
\begin{gathered}
\frac{\partial \rho}{\partial t}+\frac{\partial}{\partial s}(\rho v)=0 \\
\frac{\partial}{\partial t}(\rho v)+\frac{\partial}{\partial s}\left(\rho v^{2}\right)=\rho g_{\|}-\frac{\partial P}{\partial s} \\
\frac{\partial E}{\partial t}+\frac{\partial}{\partial s}[(E+P) v]=\rho v g_{\|}+\frac{\partial}{\partial s}\left(\kappa_{0} T^{5 / 2} \frac{\partial T}{\partial s}\right)-n^{2} \Lambda(T)+Q
\end{gathered}
$$

where

$$
E=\frac{1}{2} \rho v^{2}+\frac{P}{\gamma-1}
$$

Here, $s$ is the spatial coordinate along the loop; $n$ is the electron number density; $\rho=1.67 \times 10^{-24} \times n$ is the mass density assuming a fully ionized hydrogen plasma; $T$ is the temperature; $P=2 n k T$ is the total pressure; $v$ is the bulk velocity; $\kappa_{0}=10^{-6}$ is the coefficient of thermal conduction for Spitzer conductivity; $\gamma=5 / 3$ is the ratio of the specific heats; $g_{\|}$is the component of gravity parallel to the loop axis; $Q$ is the volumetric heating rate; and $\Lambda(T)$ is the optically thin radiative loss function as given in Klimchuk, Patsourakos, \& Cargill (2008) with the exception that there is a $T^{3}$ dependence below $0.1 \mathrm{MK}$ to account approximately for optical depth effects. 
ARGOS uses adaptive mesh refinement to dynamically modify the numerical grid in response to changes in the density gradients. This is critically important for simulations of this type. The cold condensations which form and move along the loop are bounded by thin transition regions similar to the classical transition regions at the footpoints of loops. Only by subdividing and merging grid cells is it possible to resolve these structures with a grid of reasonable size. Our simulations have approximately 3500 total cells while the condensations are present. The smallest cell length is $6 \mathrm{~km}$. This is approximately one-third the temperature scale length in the lower transition region where $T=0.1 T_{\max }$.

The loop is assumed to be a vertical semi-circle with a footpoint-to-apex half length of $L=75 \mathrm{Mm}$. This half length is characteristic of warm loops and is the value we have used for some of our nanoflare studies (e.g., Klimchuk, Patsourakos, \& Cargill 2008). The cross sectional area is constant, consistent with observations of both EUV and soft X-ray loops (Klimchuk 2000; López Fuentes, Klimchuk, \& Démoulin 2006). Attached to each end of the coronal semi-circle is a $50 \mathrm{Mm}$ chromosphere/photosphere that is maintained at a nearly constant temperature of $3 \times 10^{4} \mathrm{~K}$ by a radiative loss function that decreases precipitously to zero between $3 \times 10^{4}$ and $2.95 \times 10^{4} \mathrm{~K}$. This loss function applies to the entire loop, including the cold condensations that form in the coronal portion. Although the radiative properties of the chromosphere and condensations are treated in a highly simplified manner (there is no detailed radiative transfer), the interaction with the rest of the loop is modeled rigorously. In particular, the exchange of mass by the important processes of evaporation and condensation is fully included. Radiative transfer effects are important for explosive evaporation that is driven by energetic particle beams penetrating deep into the chromosphere during flares, but the gentle evaporation in our simulations is due to a heat flux that is mostly dissipated in the transition region. Only a small fraction of the heat flux reaches the chromosphere. 
We begin each simulation by allowing the loop to relax to a static equilibrium with a spatially uniform background heating, $Q_{b}$. The choice $Q_{b}=6 \times 10^{-4} \mathrm{erg} \mathrm{cm}^{-3} \mathrm{~s}^{-1}$ produces an apex temperature of 3.0 MK. Over the next $1000 \mathrm{~s}$, we slowly turn on a localized heating that decreases exponentially with height above the chromosphere at both ends of the loop and is spatially uniform below:

$$
Q_{l}\left(s \geq s_{0}\right)=Q_{0} \exp \left[-\left(s-s_{0}\right) / \lambda\right]
$$

on the left side, where $s_{0}=50 \mathrm{Mm}$ is the top of the chromosphere. The right side is a mirror image with the exception of amplitude (see below). Both the background and localized heating are held constant thereafter. The scale length of the exponential decrease is $\lambda=5$ $\mathrm{Mm}$, which is $1 / 15$ of the loop half length. Its maximum amplitude at the left footpoint is $Q_{0}=8.0 \times 10^{-2}$ erg $\mathrm{cm}^{-3} \mathrm{~s}^{-1}$. We impose an asymmetry by making the amplitude at the right footpoint only 50, 75, or $90 \%$ as large. The localized heating provides nearly an order of magnitude more total energy (spatially integrated over the loop) than does the uniform background heating, and therefore it dominates.

Our volumetric heating function (Eq. 5) has two broad but crucial constraints: it must be spatially localized above the chromosphere with a characteristic scale smaller than $10 \%$ of the loop length, and quasi-steady in comparison to the ambient radiative cooling time. Earlier studies of thermal nonequilibrium, as well as the physical explanation of thermal nonequilibrium (see below), all indicate that the basic phenomenon is otherwise independent of the details of the heating. Therefore, any physical heating mechanism that satisfies these constraints is capable of producing thermal nonequilibrium. Identifying which of the many candidates for coronal heating meet these criteria is an important long-term objective, but it is beyond the scope of this paper. Our sole aim is to investigate whether thermal nonequilibrium can explain ordinary coronal loops, for which purpose our heating function is appropriate. 


\section{Results}

\subsection{Monolithic Loops}

The first loop we consider is monolithic and has a $75 \%$ heating asymmetry. It exhibits quasi-periodic behavior with condensations forming roughly every 6000 s. Figure 1 shows the temperature profile at four different times during the sixth condensation cycle, long after any memory of the initial static conditions has disappeared. The evolution is typical of thermal nonequilibrium and has been well documented in our other work. After the condensation from the fifth cycle falls to the chromosphere $(t=0 \mathrm{~s})$, the loop rapidly heats and attempts to establish an equilibrium. A peak temperature of $4.4 \mathrm{MK}$ is reached at $t=650 \mathrm{~s}$. This is followed by a relatively long period of slow cooling. The solid curve in Figure 1 shows the temperature profile at $t=2950 \mathrm{~s}$, well into the cooling phase.

The reason for the slow cooling and eventual formation of a condensation can be understood as follows. We begin by noticing that the maximum temperature $T_{\text {max }}$ occurs close to the left footpoint, at a height comparable to the heating scale length $\lambda$. Let us hypothetically divide the loop into two unequal parts: a short section to the left of $T_{\max }$ and a much longer section to the right. Imagine that the short section is one-half of a small symmetric loop. If this hypothetical loop were in static equilibrium, it would satisfy the scaling law

$$
n=1.32 \times 10^{6} \frac{T_{\max }^{2}}{\ell}
$$

where $\ell$ is the half length, approximately equal to $\lambda$. Equation (6) follows from the well-known scaling law $T_{\max }=1.4 \times 10^{3}(P \ell)^{1 / 3}$ (Rosner, Tucker, \& Vaiana 1978) upon substituting for $P$ using the ideal gas law. The downward heat flux from $T_{\max }$ is very large due to the steep temperature gradient. Correspondingly large density is required in order 
for radiation from the transition region to balance the heat flux. If the actual density is smaller than the equilibrium value given in equation ([6), the radiation will be too weak, and chromospheric evaporation will occur, as it does in our simulation.

Now consider the other section of the original loop, to the right of $T_{\max }$. Imagine that it is half of a different hypothetical loop. It has the same maximum temperature as the short loop, but because it is much longer, its equilibrium density is much smaller according to equation (6). Of course the long and short "loops" are really attached. Evaporation in the short section drives up the density in the long section to values that exceed the local equilibrium conditions. Radiation is enhanced at the elevated densities, so the plasma cools.

The above argument based on the static equilibrium theory shows why static conditions are not possible with highly localized footpoint heating, but the actual energy balance in the evolving loop is more involved due to the presence of flows. The evaporating material carries an enthalpy flux that plays a very important role. It provides nearly enough energy to power the enhanced coronal radiation. This is the reason why the evolution is so slow during most of the cooling phase. In fact, if only the left leg were subjected to localized heating, the loop would establish a dynamic equilibrium with a steady end-to-end flow and no cooling (Patsourakos, Klimchuk, \& MacNeice 2004). Our loop has localized heating on both sides, which drives evaporative upflows from both ends. Because material continually accumulates in the corona, the plasma must cool, and no steady state is possible.

We see from Figure 1 that the cooling is not symmetric. Because evaporation is stronger on the left side than on the right, the flows converge to the right of the loop midpoint. Cooling is fastest at this location, and a dip develops in the temperature profile. The dip grows at an accelerating pace until a cold condensation is ultimately formed at $t=4850 \mathrm{~s}$ (dashed curve). The final collapse resembles a thermal instability; only $350 \mathrm{~s}$ are required for the temperature to drop from 2.0 to $0.03 \mathrm{MK}$. Once formed, the condensation is 
pushed to the right by a small pressure imbalance. It hits the chromosphere approximately $1300 \mathrm{~s}$ after first appearing, and a new condensation cycle begins.

\subsubsection{Excess Density Factor}

The model loop is characterized by over-dense conditions during most of its evolution. We wish to make a quantitative comparison with observations, and because many studies of observed loops involve spatial and temporal averages, we define an excess density factor $\Psi$ as follows:

$$
\Psi=\frac{\bar{n}}{\bar{n}_{e q}},
$$

where

$$
\bar{n}_{e q}=1.32 \times 10^{6} \frac{\bar{T}^{2}}{L}
$$

and $\bar{n}$ and $\bar{T}$ are the density and temperature averaged over the upper $50 \%$ of the loop and over one or more condensation cycles. Equation (8) comes from the Rosner, Tucker, \& Vaiana (1978) scaling law, analogous to equation (66). Averaging over the 11 cycles of our simulation gives $\Psi=4.09$. Note that these are simple averages using densities and temperatures taken directly from the simulation output. The excess density factor obtained in this way is different from what we would get from observed intensities, which provide nonlinear averages of density and temperature. Later we will perform a more rigorous analysis that takes this into account.

Because many observed loops are shorter or longer than our model loop, it is important to examine how $\Psi$ depends on loop length. We therefore consider two additional models that are half and twice as long as the original: $L=37.5$ and $150 \mathrm{Mm}$. The heating scale length $\lambda$ and $75 \%$ asymmetry are the same as before, but we modify the heating amplitudes 
$Q_{b}$ and $Q_{0}$ so that the peak temperatures of the initial equilibrium and of the condensation cycles are similar in all three cases. The resulting excess density factors are 2.90 and 6.62 for the short and long loops, respectively.

These three cases suggest the relationship $\Psi \propto L^{1 / 2}$. We can understand the square-root dependence by considering the period of slow cooling that dominates the evolution. As discussed above, a strong downward heat flux drives an upward enthalpy flux in the lower legs:

$$
\kappa_{0} \frac{T_{m a x}^{7 / 2}}{\lambda} \approx \frac{5}{2} P v
$$

The enthalpy is nearly enough to power the radiative losses from the rest of the loop:

$$
\frac{5}{2} P v \approx n^{2} \Lambda\left(T_{\max }\right) L .
$$

Combining, we get

$$
n \approx\left[\frac{\kappa_{0} T_{\max }^{7 / 2}}{\Lambda\left(T_{\max }\right)} \frac{1}{\lambda L}\right]^{1 / 2}
$$

for the actual loop density.

In static equilibrium, the energy loss rates from radiation and thermal conduction are approximately equal in the corona (Vesecky, Antiochos, \& Underwood 1979):

$$
n_{e q}^{2} \Lambda\left(T_{\max }\right) \approx \kappa_{0} \frac{T_{\max }^{7 / 2}}{L^{2}} .
$$

This gives 


$$
n_{e q} \approx\left[\kappa_{0} \frac{T_{\max }^{7 / 2}}{\Lambda\left(T_{\max }\right)} \frac{1}{L^{2}}\right]^{1 / 2}
$$

for the equilibrium density corresponding to $T_{\max }$ and $L 1$ The excess density factor is therefore

$$
\Psi=\frac{n}{n_{e q}} \approx\left(\frac{L}{\lambda}\right)^{1 / 2}
$$

Note that it depends not on the loop length alone, but on the ratio of the loop length to heating scale length. In principle, we could reproduce model loops with any $L$ and $\Psi$ simply by adjusting the value of $\lambda$. It seems, therefore, that the observed excess densities of warm loops can be readily explained with thermal nonequilibrium.

\subsubsection{Intensity}

A successful loop model must also explain the intensity properties of observed loops, both temporal and spatial. We therefore generate light curves and intensity profiles for simulated TRACE observations of the models made in the 171 channel. We assume that the loops are viewed from the side, so the intensity at any point along the loop axis is given by $I=n^{2} G(T)$. Here, $G(T)$ is the instrument response function, which for the 171 channel is reasonably sharply peaked near $1 \mathrm{MK}$. We have ignored the loop diameter and a possible filling factor because we are concerned only with relative intensities, and both the diameter and filling factor are assumed to be constant along the loop and unchanging in time.

The solid curve in Figure 2 is the light curve for the sixth condensation cycle of the original $L=75 \mathrm{Mm}$ loop. This is the same condensation cycle shown in Figure 1) We

\footnotetext{
${ }^{1}$ Comparing equations 13 and 8, we see that the Rosner, Tucker, \& Vaiana scaling law uses $\Lambda(T) \propto T^{-1 / 2}$.
} 
have averaged the intensity over the upper $80 \%$ of the loop to exclude the "moss" emission from the transition regions at the footpoints. The dashed and dotted curves show the corresponding evolution of the spatially averaged temperature and density. It is readily apparent how evaporation slowly fills the loop with plasma.

Before the condensation forms, the coronal plasma is too hot to be easily detected in the 171 channel, and the light curve is extremely faint. It brightens dramatically when the condensing plasma cools rapidly through $1 \mathrm{MK}$ (sharp spike at $t=4700 \mathrm{~s}$ ). This contrasts with the much more gradual brightening exhibited by most observed loops (Winebarger, Warren, \& Seaton 2003). The light curve remains bright after the condensation has fully formed because transition regions are present on either side of the cold mass. After about $1000 \mathrm{~s}$ the light curve suddenly dims as the condensation moves out of the $80 \%$ averaging window. The spatially-averaged density drops at the same time since the condensation contains most of the loop's mass. Bright emission is actually present in the loop for another $300 \mathrm{~s}$ as the condensation traverses the remaining $20 \%$ of the leg before hitting the chromosphere. The total lifetime in 171 emission is therefore approximately $1300 \mathrm{~s}$. This is at the extreme low end of the range of observed lifetimes.

The abrupt appearance and disappearance of the 171 emission disagrees with observations, which show a more gradually evolving light curve. The spatial distribution of the emission presents an even bigger problem. Figure 3 shows profiles of intensity (solid) and temperature (dashed) at $t=5000 \mathrm{~s}$, after the condensation has formed. The emission is highly concentrated in transition region layers at the loop footpoints ("moss") and on either side of the condensation. This contrasts sharply with observed loops, which tend to have a far more uniform appearance. Falling bright knots are sometimes observed, but these are only detected at much cooler temperatures ( $\leq 0.1 \mathrm{MK}$ ) (Schrijver 2001; De Groof et al. 2004; O'Shea, Banerjee. \& Doyle 2007). We return to the subject of these knots later in the 
Discussion section.

To determine whether the extreme nonuniformity in the intensity distribution is affected by the degree of heating asymmetry, we perform two additional simulations using the same heating amplitude and scale length as before, but with asymmetries of $50 \%$ and 90\%. The results are qualitatively similar to the $75 \%$ case. The intensity profiles are highly structured and in gross disagreement with observations.

The primary reason for the nonuniform intensity is that most of the loop is too hot to be easily detected in the 171 channel (i.e., significantly hotter than $1 \mathrm{MK}$ ). To obtain temperatures more suitable to 171 , we perform three new simulations with the heating amplitude reduced by an order of magnitude. All other parameters are as before. The model with $75 \%$ asymmetry reaches a maximum temperature of $1.8 \mathrm{MK}$ and has an excess density factor $\Psi=4.69$. The results for the $50 \%$ and $90 \%$ cases are similar.

Figure 4 shows the 171 light curve and the temperature and density evolution for the second condensation cycle of the $75 \%$ case. The cycle lasts approximately 11,000 s, nearly twice as long as the strong heating counterpart. The light curve has three rather distinct phases - faint, bright, and intermediate - which is not consistent with the slowly varying intensities of most observed warm loops. The bright and intermediate phases together last about $7000 \mathrm{~s}$, which is longer than most observed loop lifetimes.

An interesting aspect of this simulation is that two condensations are present at the same time, as was seen in earlier studies (Müller. Peter, \& Hansteen 2004; Karpen et al. 2005). Figures 5 and 6 show intensity and temperature profiles at $t=5000$ and $7000 \mathrm{~s}$, before and after the condensations form. More of the loop is visible than in the strong heating models, but the intensity still is far more structured than is observed. In particular, the region between the condensations is extremely faint. We can understand this behavior as follows. When the condensations form, the central region between them is cut off from 
the evaporative upflows and associated enthalpy flux that powers the radiative losses. The plasma cools and drains onto the condensations. The condensations behave like chromospheres, and a quasi-static loop equilibrium is established between them. Because the heating rate is so small, the equilibrium state has a low temperature and very low density, so the 171 emission is minimal. The precise value of the temperature and density depend on the magnitude of the uniform background heating, which dominates in this part of the loop. Note that the two condensations remain separate at all times and do not merge, as is sometimes seen in other simulations (e.g., Karpen et al. 2005).

Thermal nonequilibrium clearly cannot explain observed loops if the loops are monolithic structures, at least not with steady, exponential heating of the type we have considered.

\subsection{Multi-Strand Loops}

\subsubsection{Excess Density Factor}

Because our monolithic models fail, we now consider loops that are bundles of many unresolved strands. To start, we assume that all of the strands in a given loop are identical except for the phasing of the condensation cycles, which we take to be random. We can then approximate an instantaneous snapshot of the composite loop by simply time averaging one simulation over one or more cycles.

A wide variety of temperatures coexist within the cross section of such a multi-stranded loop. The single temperature that is measured by an instrument like TRACE or $\mathrm{SXT} /$ Yohkoh is a weighted average, where the weighting depends on both the temperature response function, $G(T)$, and the differential emission measure distribution, $D E M(T)$. To simulate realistic measurements from our models, we first compute intensity profiles for 
the individual strands (i.e., for each time in the simulation), and then we average them together to obtain a single intensity profile for the loop bundle. We do this separately for the 171 and 195 channels of TRACE and the Al.1 and AlMg channels of SXT. We next infer temperature and emission measure, $E M$, at each position along the loop using both the 171/195 and Al.1/AlMg ratios. From the emission measure, we compute density according to

$$
n=\left(\frac{E M}{d f}\right)^{1 / 2}
$$

where $d$ is the loop diameter and $f$ is the filling factor. The diameter plays no role, since $E M$ is derived from the loop intensity, which scales with the assumed diameter. We take a filling factor of unity, precisely as done for real data, which means that the density given by equation (15) is a lower limit. Finally, we average $T$ and $n$ along the upper $50 \%$ of the loop 2 and use equations (17) and (8) to obtain the excess density factors that would be measured by TRACE and SXT, designated $\Psi_{T R A C E}$ and $\Psi_{S X T}$. We follow this procedure separately for all 6 of the $L=75 \mathrm{Mm}$ models (2 heating amplitudes and 3 heating asymmetries).

It seems unlikely that all of the strands in a given loop bundle would be identical except for their phases. Therefore, we also build a composite loop with strong heating and a composite loop with weak heating by averaging together the models with 50, 75, and 90\% asymmetry. The averages include both the original models, which have greater heating in the left leg, and their mirror images, which have greater heating in the right leg. The two composite loops so obtained have a mixture of strands of different types, which is perhaps more realistic. We simulate temperature and density measurements of these loops using the

\footnotetext{
${ }^{2}$ The reader may wonder why we use $50 \%$ averages here and $80 \%$ averages for the light curves. $50 \%$ was used in the observational studies of loop density to which we will compare our models. For the light curves, we are only concerned with excluding the bright moss emission at the footpoints.
} 
same procedure described above, first averaging the intensities and then applying the filter ratio technique.

Results for the "homogeneous" multi-strand models and the composite multi-strand models are presented in Table 1. The first column gives the loop half length, which is the same for all except the last two cases. The second column gives the amplitude of the localized heating together with an indication of whether it is strong (produces a peak temperature near 4.4 MK) or weak (produces a peak temperature near 1.8 MK). The third column gives the heating asymmetry. The fourth column gives the number of condensation cycles used in the temporal averages. The fifth column gives the average period of the cycles, which are only quasi-regular in most cases. The sixth and seventh columns give the temperatures that would be measured with TRACE and SXT filter ratios. The last three columns give the excess density factors obtained directly from the temperatures and densities of the models, equation (7), and from the simulated TRACE and SXT measurements. The values differ because TRACE preferentially detects the warm plasma and SXT preferentially detects the hot plasma.

Figure 7 shows the excess density factors of real loops plotted against temperature. The factors were determined precisely as described above, i.e., using equation (15). The loops near $1 \mathrm{MK}$ (pluses) were observed by TRACE and analyzed originally by Aschwanden, Nightingale, \& Alexander (2000). The hotter loops (crosses) were observed by SXT and analyzed originally by Porter \& Klimchuk (1995) (also Klimchuk \& Porter 1995). These are the same loops presented in Figure 4 of Winebarger, Warren, \& Mariska (2003) and Figure 6 of Klimchuk (2006). Also shown are the excess density factors of the model loops as determined from simulated TRACE observations (diamonds) and simulated SXT observations (squares).

There is good agreement between the models and observations for the excess density 
factors obtained from TRACE. Values range between about 3 and 11 for the models and between about 1 and 12 for the observations (note that logarithms are plotted in the figure). The temperatures measured by $T R A C E$ are also in good agreement, but this is expected because the 171 and 195 filters have a narrow temperature response and are only sensitive to plasma close to $1 \mathrm{MK}$.

The agreement between the models and observations is much worse for the SXT measurements. Excess density factors from the models are tightly clustered between 1 (no excess) and 3, whereas those from the observations range all the way from 0.02 (highly under dense) to 16 . The agreement is better if we restrict ourselves to the temperature range of the models $\left(1.1<T_{S X T}<3.4 \mathrm{MK}\right)$, in which case the observed excess density factors are all $>0.4$ (slightly under dense). However, the observed factors have a strong tendency to decrease with temperature, while the model factors have a weak tendency to increase. We conclude that the models are consistent with at most a subset of observed SXT loops. According to equation (14), thermal nonequilibrium can never produce the under-dense conditions observed at high temperatures because $\lambda>L$ gives rise to static equilibrium (in fact, static equilibrium occurs whenever $\lambda>L / 5$ approximately). It is worth pointing out that the nanoflare storm model is capable of explaining both over-dense warm loops and under-dense hot loops (Klimchuk 2006).

The quantity $\Psi$ defined in equations (77) and (8) very likely underestimates the true degree to which TRACE loops are over-dense (cf. Winebarger, Warren, \& Mariska 2003). Equation (8) is an idealized scaling law based on: (1) an approximate and somewhat outdated form for the radiative loss function; (2) the assumption of no gravitational stratification; and (3) the assumption of spatially uniform heating. The coefficient of the scaling law should be treated with particular caution. Furthermore, the "actual" density determined from equation (15) assumes a filling factor $f=1$ and is therefore a lower limit, 
but the equilibrium density determined from equation (8) does not depend on $f$. Despite these limitations, $\Psi$ is a useful tool for evaluating the agreement between models and observations.

\subsubsection{Intensity and Temperature}

We rejected the monolithic models on the basis of their 171 intensity properties, and we now examine whether the multi-strand models fare any better. We limit our discussion to the composite models because we believe they are more realistic and, more importantly, because they agree better with the observations.

The biggest failing of the monolithic models is their highly structured intensity profile. The problem is especially severe for models with strong heating, which have localized bright emission immediately flanking the cold condensation. Multi-strand models have a much more uniform appearance because they include many unresolved condensations that are spread out along the loop. Condensations tend to form in the upper two-thirds of the loop, at a location that depends on the level of heating asymmetry and on $\lambda$. The weaker the asymmetry, the closer to the apex they form, with perfectly symmetric heating producing a condensation right at the apex. Once formed, the condensations move downward toward the footpoints. If all phases of the cycles are represented in the strands, the entire loop will be filled in with bright emission, including the lower legs, consistent with observations. It is critical, however, that some of the strands have nearly symmetric heating so that a dark gap is not present at the top.

Figure 8 shows the intensity profile for the composite model with strong heating. Except for the bright spikes at the footpoints (note the logarithmic scale), the emission is reasonably uniform. Intensity variations along the loop are less than a factor of 2 and 
would be smaller still if the bundle included a larger variety of heating asymmetries.

Figure 9 shows the 171 intensity profile for the composite model with weak heating (linear scale). The profile is very smooth, due largely to the fact that the individual strands are reasonably uniform up to the time when the condensations form. The profile is nonetheless inconsistent with observations because the intensity decreases too rapidly with height. The scale height in the model corresponds to a hydrostatic loop at $1 \mathrm{MK}$, whereas observed scale heights are super-hydrostatic by up to a factor of 2 .

Figure 10 shows three temperature profiles for the strong heating composite model. The solid curve is the average of the actual temperatures in the strands; the dashed curve is the temperature that would be measured by TRACE; and the dotted curve is the temperature that would be measured by SXT. The temperature profiles are very flat, in excellent agreement with TRACE observations and not inconsistent with SXT observations (Kano \& Tsuneta 1996). The composite model with weak heating also has a flat TRACE temperature profile. Its SXT profile is not relevant, since the loop would be extremely faint in soft X-rays.

The multi-strand models presented here were obtained by temporally averaging over two or more condensation cycles. As such, they represent very long-lived loops, inconsistent with observations. We could instead average over a portion of a cycle to obtain a shorter lived loop, but then the intensity and temperature profiles would be less uniform. Averaging over a portion of the cycle corresponds to condensations forming at roughly the same time in the different strands. If they form at the same time, they move together as a group. The lower legs of such a loop would be dark in the early stages of evolution, and the apex would be dark in the later stages, neither of which agrees with observations. Whether it is possible to build a loop that is both sufficiently short lived and sufficiently uniform to match observed loops is a question that we examine in more detail below. 


\section{Discussion and Conclusions}

We have modeled monolithic and multi-strand loops undergoing thermal nonequilibrium with the hope of reproducing the salient features of observed loops, especially those seen in warm $(\sim 1 \mathrm{MK})$ emissions by instruments like TRACE. A fundamental property of these warm loops is their excess density compared to static equilibrium. We find that many of our models can successfully explain the observed densities. Some can also explain the unstructured intensities and flat temperature profiles that are typically observed. However, none of the models is able to successfully reproduce all of the observed properties. The monolithic models fail dramatically in that they have far too much intensity structure. This is not a problem for the multi-strand models, but these models, as presented, are far too long-lived. The competing requirements of uniform intensity and short-to-modest lifetime (1000-5000 s) are extremely difficult to satisfy. It may be possible to construct a model that satisfies both, but the conditions are too contrived to be a plausible explanation for real loops, as we now show.

It is instructive to briefly discuss the nanoflare storm concept (Klimchuk 2009), because it shares several common features with the thermal nonequilibrium scenario we are now considering. In the nanoflare case, each loop is envisioned to be a bundle of strands that are heated impulsively at different times (but only once). At any given moment, the many strands are in different stages of cooling and therefore only some of them are detectable in the 171 channel. If the duration of the nanoflare storm (time delay between the first and last nanoflare) is long compared to the lifetime of each strand (duration of visibility), then the lifetime of the entire loop bundle will be determined primarily by the duration of the storm. If, on the other hand, the duration of the storm is short compared to the lifetime of each strand, then the lifetime of the bundle will be determined primarily by the lifetime of the strands. It is straightforward to see that the loop lifetime is approximately equal to the 
sum of the storm duration and the strand lifetime.

We can apply these same ideas to a bundle of strands undergoing thermal nonequilibrium. In place of the nanoflare storm duration, we have the time delay $\Delta t$ between the formation of the first and last condensations. Just as there is only one nanoflare per strand, there can be only one condensation (or condensation pair) per strand, because the period of the cycles is considerably longer than observed loop lifetimes. Let $\tau$ represent the time that each strand is visible in the 171 channel. To reproduce the observed loop lifetimes, $\Delta t$ must satisfy $\Delta t+\tau \approx 1000-5000$ s. Model strands with weak heating have $\tau>5000$ and can be immediately ruled out. Model strands with strong heating have $\tau \approx$ 1000-2000 s (2000 s for the case with 90\% heating asymmetry). Observed loop lifetimes can perhaps be reproduced if $\Delta t \approx 0-4000 \mathrm{~s}$.

The condition on $\Delta t$ is necessary but not sufficient. Loops will have uniform intensity only if the strands are sufficiently out of phase. There is a problem when $\Delta t$ is small because then all of the strands are roughly in phase. The condensations form together in the upper part of the loop and move together down the leg. The requirement of uniform brightness places a lower limit on $\Delta t$ that is approximately the time it takes a single condensation to traverse the entire half length of the loop. Only then will one condensation appear near the apex at the same time that another is about to disappear into the chromosphere. In the simulation with $90 \%$ heating asymmetry, the condensation takes approximately $2000 \mathrm{~s}$ to traverse this distance. This is also how long the strand is visible in 171 . To have a uniform loop bundle made from these strands implies a loop lifetime of at least $\Delta t+\tau \approx 2000+2000=4000$ s. A majority of observed warm loops are shorter lived than this. We conclude that they cannot be explained by thermal nonequilibrium.

Even the longer lived loops are problematic. To produce a condensation, the heating in each strand must be steady or quasi-steady for at least one cycle, which lasts approximately 
2 hours. If the heating is steady for 2 hours, then it seems reasonable to expect that it might remain steady for 4 hours, or even longer. This would allow additional cycles to occur and the loop to reappear multiple times. We can rule this out, however. As shown in Table 1, strands with different heating parameters have different cycle periods. The period also varies from one cycle to the next for a given set of parameters (i.e., the cycles are only quasi-periodic). Therefore, even if the phasing of the strands were correct for the first appearance of the loop - itself a challenge - it would not be correct for the second and subsequent appearances. To reproduce the observations, the heating must turn on, remain steady for one full cycle, and then turn off before any new condensations can form. This seems highly implausible. We conclude that thermal nonequilibrium is not a reasonable explanation for any observed warm coronal loops, even those that are relatively long-lived. Thermal nonequilibrium is also incapable of explaining hot loops, since it cannot produce the under-dense conditions that are characteristic of these loops.

An important implication of our results is that the dominant heating mechanism in active regions cannot be both highly concentrated low in the corona and steady or quasi-steady (slowly varying or impulsive with a rapid cadence). Active regions would look much different if this were the case. Loops resembling our models - and therefore unlike those observed - would be common. This claim must be qualified with some caveats. It is acceptable for the heating to decrease with height as long as the scale length is greater than about $20 \%$ of the loop half length. Only shorter scale lengths produce thermal nonequilibrium. Even these short lengths might be allowed if only one leg of the loop is heated, because then a steady flow equilibrium can be established. It is unclear, however, whether these steady equilibria can reproduce the excess densities, intensity scale heights, and temperature profiles that are observed (Patsourakos, Klimchuk, \& MacNeice 2004; Winebarger et al. 2002). 
Finally, we cannot exclude the possibility that thermal nonequilibrium is occurring in the diffuse corona between loops. The properties of this part of the corona are not well understand, and evidence of thermal nonequilibrium might not be obvious if there is a multitude of unresolved strands with random phasing of the cycles. One consequence of many unresolved condensations would be the absorption of the EUV radiation from below. Evidence of absorption from unresolved cold material in the corona has been reported (e.g., Schmahl \& Orrall 1979; Klimchuk \& Gary 1995), but whether the quantities are consistent with widespread thermal nonequilibrium has not yet been investigated.

We close by emphasizing that thermal nonequilibrium is likely to play an important role in the solar atmosphere under more limited circumstances. It is almost certainly responsible for prominences (Antiochos \& Klimchuk 1991; Karpen, Antiochos, \& Klimchuk 2006), and it may also explain "catastrophic cooling events," including coronal rain (Schrijver 2001; De Groof et al. 2004; O'Shea, Baneriee, \& Doyle 2007). During these events, a cold "blob" condenses out of the hot corona at the top of a loop. It appears sequentially in the 195, 171, 1600, and 1216 channels of TRACE, which have maximum sensitivity at temperatures of 1.5, 1.0, 0.1, and 0.02 MK, respectively. The blob is visible only in the two coolest channels as it falls down the leg at speeds of $20-100 \mathrm{~km} \mathrm{~s}^{-1}$. Though fascinating, these events are relatively uncommon. The number of blobs observed at any one time is much less than the number of warm loops (C. Schrijver, 2009, private communication).

Müller, Peter, \& Hansteen (2004) and Antolin \& Shibata (2009) have suggested that these blobs are formed by thermal nonequilibrium. Our monolithic models with strong heating have many similarities to the observations, including the downward velocities, but key differences are not yet explained. The observed time delay between the blob's appearance in the 171 and 1600 channels is more than twice what our models predict. More significantly, our models predict 171 and 195 emission from the transition regions that flank 
the blob as it falls, but such emission is apparently not seen. It is clear that more work is needed before we fully understand the origin of catastrophic cooling events.

This work was supported by the NASA Living With a Star program. A portion of it was performed while the authors were on the staff of the Naval Research Laboratory. We gratefully acknowledge useful conversations with Roberto Lionello, Jon Linker, Yung Mok, Karel Schrijver, and Daniele Spadaro.

Table 1: Model Parameters

\begin{tabular}{cccccccccc}
\hline$L$ & $Q_{0}$ & Asymmetry & Cycles & Period & $T_{T R A C E}$ & $T_{S X T}$ & $\Psi$ & $\Psi_{T R A C E}$ & $\Psi_{S X T}$ \\
{$[\mathrm{Mm}]$} & {$\left[\mathrm{erg} \mathrm{cm}^{-3} \mathrm{~s}^{-1}\right]$} & {$[\%]$} & & {$[\mathrm{s}]$} & {$[\mathrm{MK}]$} & {$[\mathrm{MK}]$} & & & \\
\hline 75 & $8.0 \times 10^{-2}$ (strong) & 90 & 9 & 7330 & 1.30 & 3.35 & 5.07 & 9.69 & 3.00 \\
75 & $8.0 \times 10^{-2}($ strong $)$ & 75 & 11 & 6370 & 1.31 & 3.16 & 4.09 & 6.79 & 3.15 \\
75 & $8.0 \times 10^{-2}($ strong $)$ & 50 & 2 & 6800 & 1.36 & 3.15 & 3.56 & 4.74 & 3.02 \\
75 & $8.0 \times 10^{-2}$ (strong) & Composite & $2-11$ & & 1.24 & 3.24 & 4.26 & 8.88 & 3.01 \\
75 & $8.0 \times 10^{-3}$ (weak) & 90 & 2 & 10,280 & 1.20 & 1.65 & 2.73 & 2.42 & 1.25 \\
75 & $8.0 \times 10^{-3}$ (weak) & 75 & 2 & 10,280 & 1.19 & 1.47 & 4.69 & 2.53 & 1.52 \\
75 & $8.0 \times 10^{-3}$ (weak) & 50 & 3 & 6370 & 1.06 & 1.11 & 5.18 & 3.66 & 2.99 \\
75 & $8.0 \times 10^{-3}$ (weak) & Composite & $2-3$ & & 1.14 & 1.51 & 4.05 & 2.85 & 1.45 \\
150 & $2.0 \times 10^{-2}$ (strong) & 75 & 3 & 7700 & 1.22 & 3.37 & 6.62 & 11.34 & 3.10 \\
37.5 & $3.2 \times 10^{-1}$ (strong) & 75 & 5 & 6110 & 1.29 & 3.28 & 2.90 & 4.98 & 2.35 \\
\hline
\end{tabular}




\section{REFERENCES}

Antiochos, S. K., \& Klimchuk, J. A. 1991, ApJ, 378, 372

Antiochos, S. K., MacNeice, P. J., Spicer, D. S.,\& Klimchuk, J. A. 1999, ApJ, 512, 985

Antolin, P., \& Shibata, K. 2009, ApJ, submitted

Aschwanden, M. J., Nightingale, R. W., \& Alexander, D. 2000, ApJ, 541, 1059

Aschwanden, M. J., Schrijver, C. J., \& Alexander, D. 2001, ApJ, 550, 1036

Aschwanden, M. J., Newmark, J. S., Delaboudiniere, J. P., Neupert, W. M., Klimchuk, J. A., Gary, G. A., Portier-Fornazzi, F., \& Zucker, A. 1999, ApJ, 515, 842

De Groof, A., Berghmans, D., van Driel-Gesztelyi, L., \& Poedts, S. 2004, å, 415,1141

Kano, R. \& Tsuneta, S. 1996, PASJ, 48, 535

Karpen, J. T. \& Antiochos, S. K. 2008, ApJ, 676, 658

Karpen, J. T., Antiochos, S. K., Hohensee, M., Klimchuk, J. A., \& MacNeice, P. J. 2001, ApJ(Lett), 553, L85

Karpen, J. T., Antiochos, S. K., \& Klimchuk, J. A. 2006, ApJ, 637, 531

Karpen, J. T., Antiochos, S. K., Klimchuk, J. A., \& MacNeice, P. J. 2003, ApJ, 593, 1187

Karpen, J. T., Antiochos, S. K., Tanner, S. E. M., \& DeVore, C. R. 2005, ApJ, 635, 1319

Klimchuk, J. A. 2000, Sol. Phys., 193, 53

Klimchuk, J. A. 2006, Sol. Phys., 234, 41 
Klimchuk, J. A. 2009, in ASP Conf. Ser. XX, Proceedings of the Second Hinode Science Meeting: Beyond Discovery-Toward Understanding, eds. M. Cheung, B. Lites, T. Magara, J. Mariska, \& K. Reeves (San Francisco: Astron. Soc. Pacific)

Klimchuk, J. A., \& Gary, D. E. 1995, ApJ, 448, 925

Klimchuk, J. A., Patsourakos, S., \& Cargill, P. J. 2008, ApJ, 682, 1351

Klimchuk, J. A., \& Porter, L. J. 1995, Nature, 377, 131

Lenz, D. D., DeLuca, E. E., Golub, L., Rosner, R., \& Bookbinder, J. A. 1999, ApJ, 517, L15

López Fuentes, M. C., Démoulin, P., \& Klimchuk, J. A. 2008, ApJ, 673, 586

López Fuentes, M. C., Klimchuk, J. A., \& Démoulin, P. 2006, ApJ, 639, 459

López Fuentes, M. C., Klimchuk, J. A., \& Mandrini, C. H. 2007, ApJ, 657, 1127

Mok, Y., Mikić, Z., Lionello, R., \& Linker, J. A. 2008, ApJ(Lett), 679, L161

Müller, D. A. N., Hansteen, V. H., \& Peter, H. 2003, å, 411, 605

Müller, D. A. N., , Peter, H., \& Hansteen, V. H. 2004, å, 424, 289

O’Shea, E., Banerjee, D., \& Doyle, J. G. 2007, å, 475, L25

Patsourakos, S., Klimchuk, J. A., \& MacNeice, P. J. 2004, ApJ, 603, 322

Porter, L. J., \& Klimchuk, J. A. 1995, ApJ, 454, 499

Reale, F., \& Peres, G. 2000, ApJ(Lett), 528, L45

Rosner, R., Tucker, W. H., \& Vaiana, G. S. 1978, ApJ, 220, 643

Schmahl, E. J., \& Orrall, F. Q. 1979, ApJ(Lett), 231, L41 
Schriver, C. J. 2001, Sol. Phys., 198, 325

Susino, R., Lanzafama, A. C., Lanza, A. F., \& Spadaro, D. 2010, ApJ, 709, 499

Testa, P., Peres, G., \& Reale, F. 2005, ApJ, 622, 695

Ugarte-Urra, I., Warren, H. P., \& Brooks, D. H. 2009, ApJ, 695, 642

Ugarte-Urra, I., Winebarger, A. R., \& Warren, H. P. 2006, ApJ, 643, 1245

Vesecky, J. F., Antiochos, S. K., \& Underwood, J. H. 1979, ApJ, 233, 987

Winebarger, A. R., \& Warren, H. P. 2005, ApJ, 626, 543

Winebarger, A. R., Warren, H. P., \& Mariska, J. T. 2003, ApJ, 587, 439

Winebarger, A. R., Warren, H. P., \& Seaton, D. B. 2003, ApJ, 593, 1164

Winebarger, A. R., \& Warren, H., van Ballegooijen, A., DeLuca, E. E., \& Golub, L. 2002, ApJ, 567, L89 


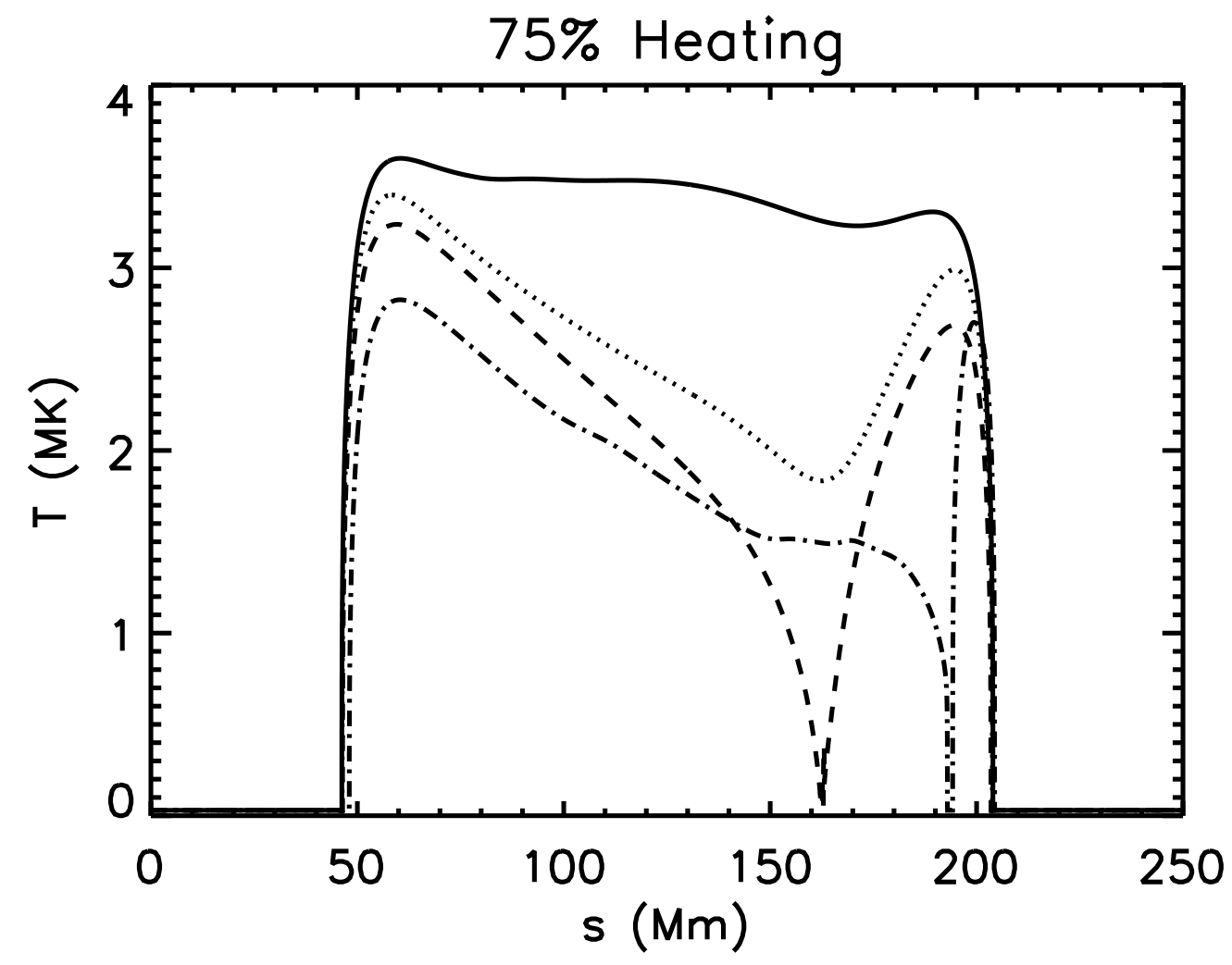

Fig. 1.- Temperature versus position at four times during the sixth condensation cycle of the loop with strong heating and 75\% asymmetry: $t=2950$ (solid), 4500 (dotted), 4850 (dashed), and 5750 (dot-dashed) seconds after the previous condensation hits the chromosphere. 


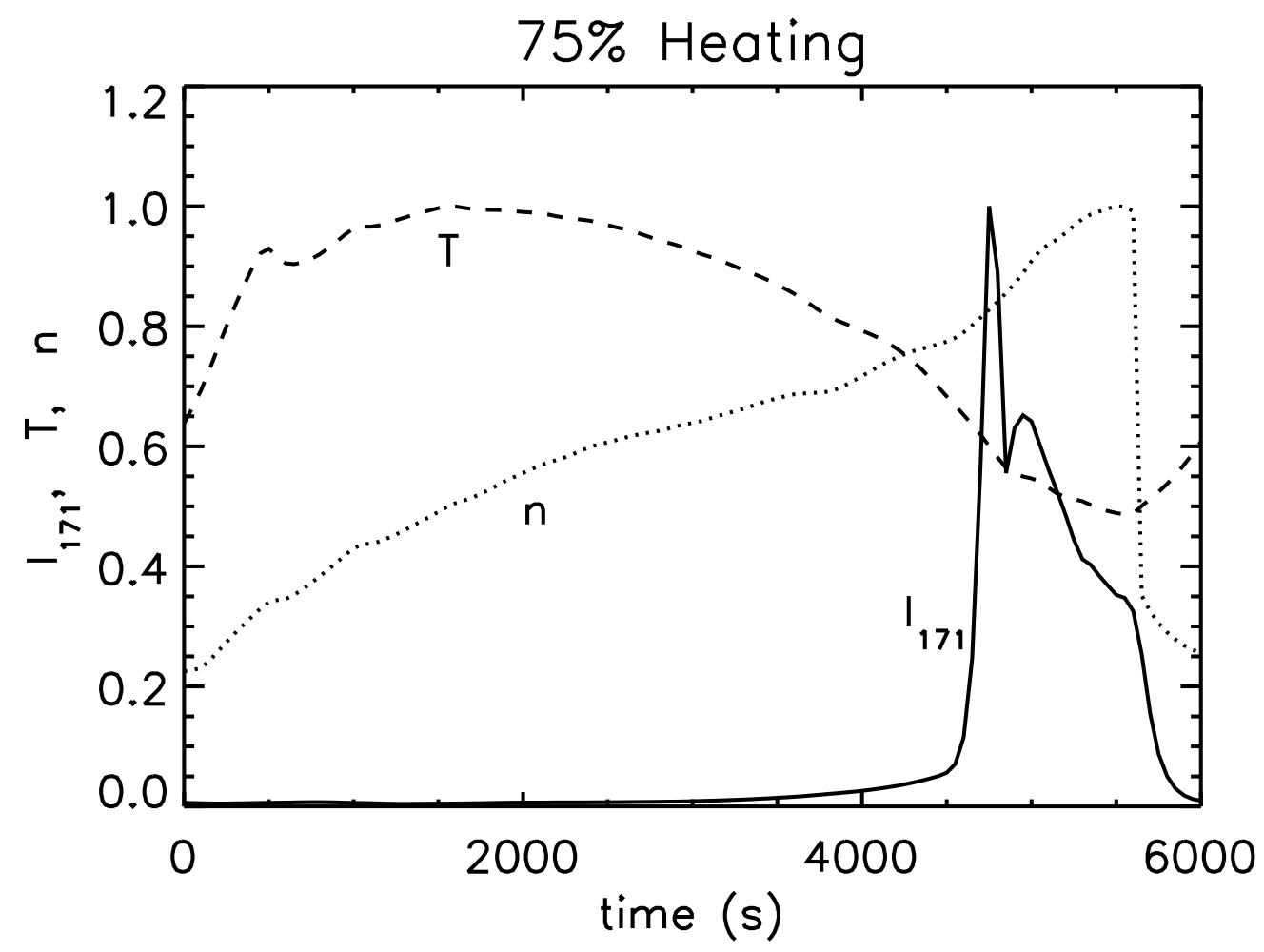

Fig. 2.- TRACE 171 intensity (solid), temperature (dashed), and density (dotted) versus time for the sixth condensation cycle of the loop with strong heating and $75 \%$ asymmetry. Values are averaged over the upper $80 \%$ of the loop and are normalized to their respective maxima. 


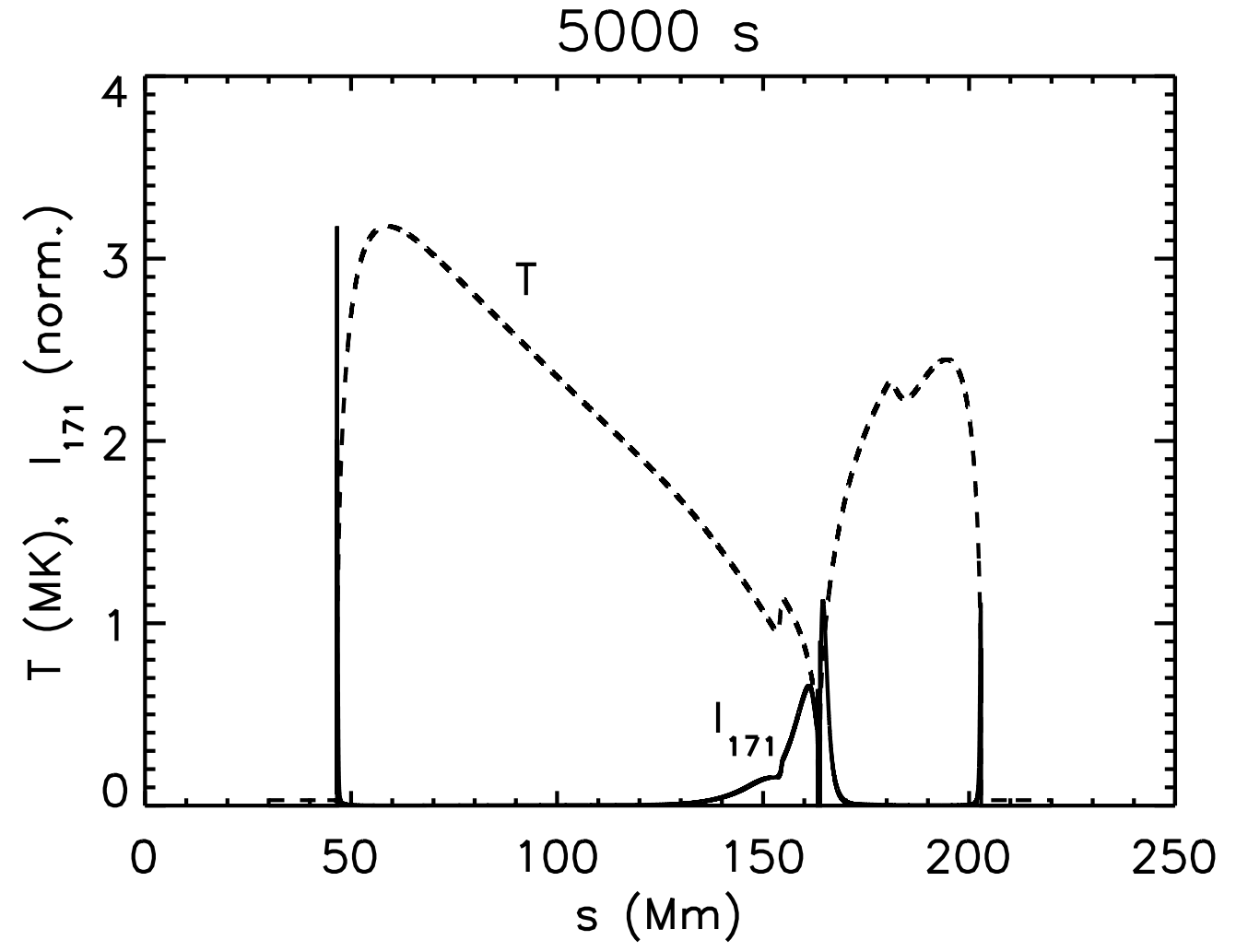

Fig. 3.- TRACE 171 intensity (solid) and temperature (dashed) versus position at $t=5000$ $\mathrm{s}$ in the sixth condensation cycle of the loop with strong heating and $75 \%$ asymmetry. 


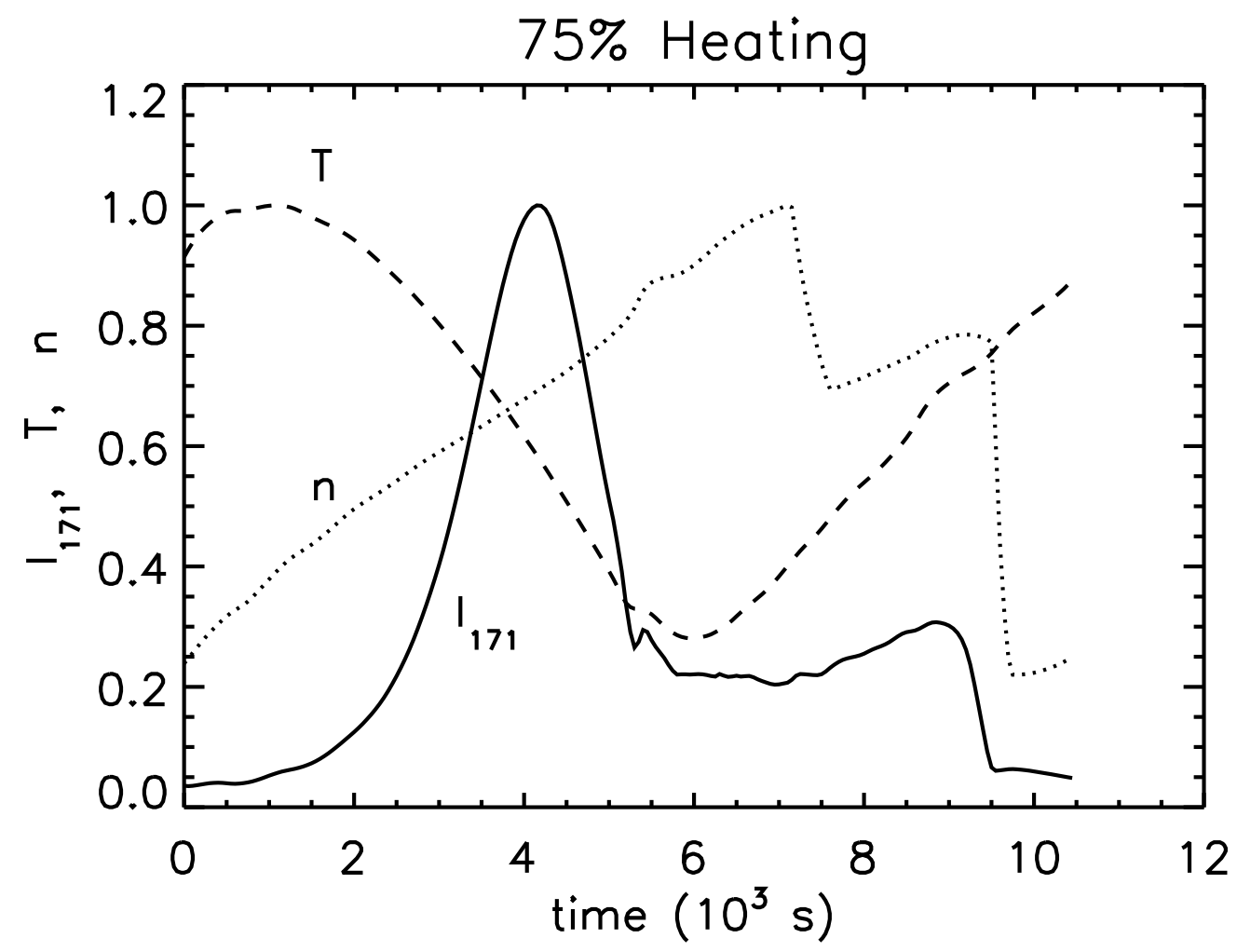

Fig. 4.- TRACE 171 intensity (solid), temperature (dashed), and density (dotted) versus time for the second condensation cycle of the loop with weak heating and $75 \%$ asymmetry. Values are averaged over the upper $80 \%$ of the loop and are normalized to their respective maxima. 


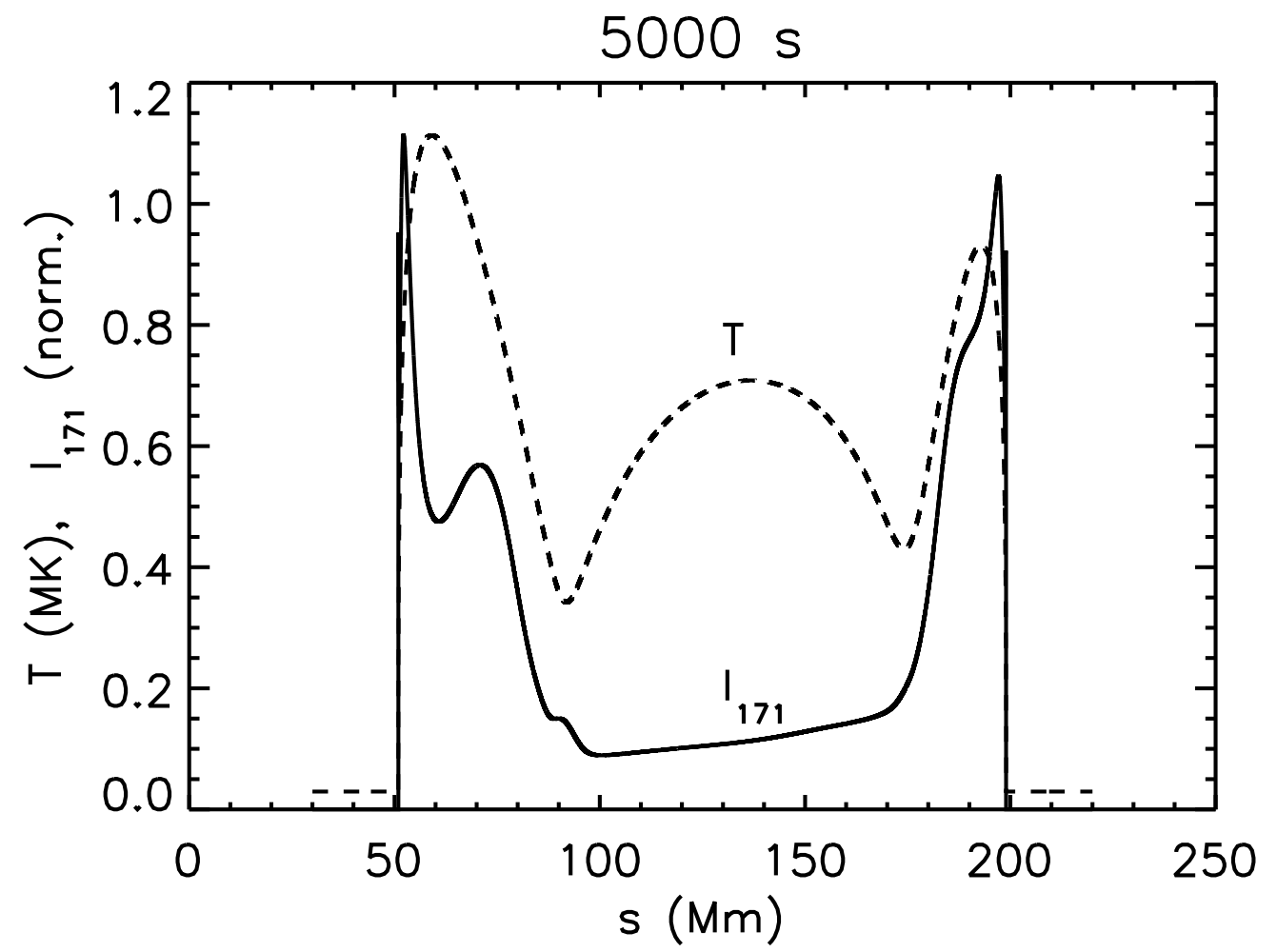

Fig. 5. - TRACE 171 intensity (solid) and temperature (dashed) versus position at $t=5000$ $\mathrm{s}$ in the second condensation cycle of the loop with weak heating and $75 \%$ asymmetry. 


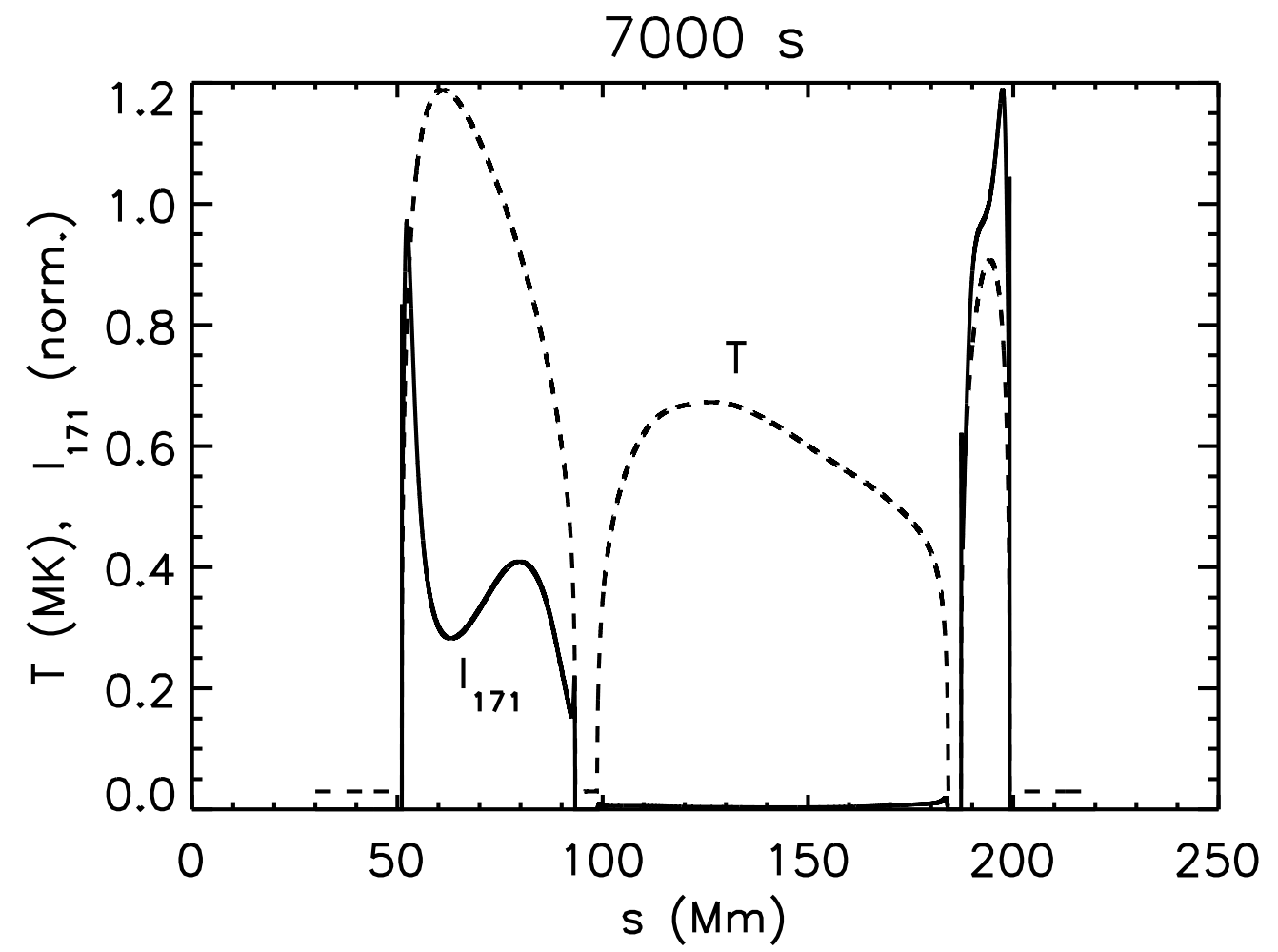

Fig. 6. - TRACE 171 intensity (solid) and temperature (dashed) versus position at $t=7000$ $\mathrm{s}$ in the second condensation cycle of the loop with weak heating and $75 \%$ asymmetry. 


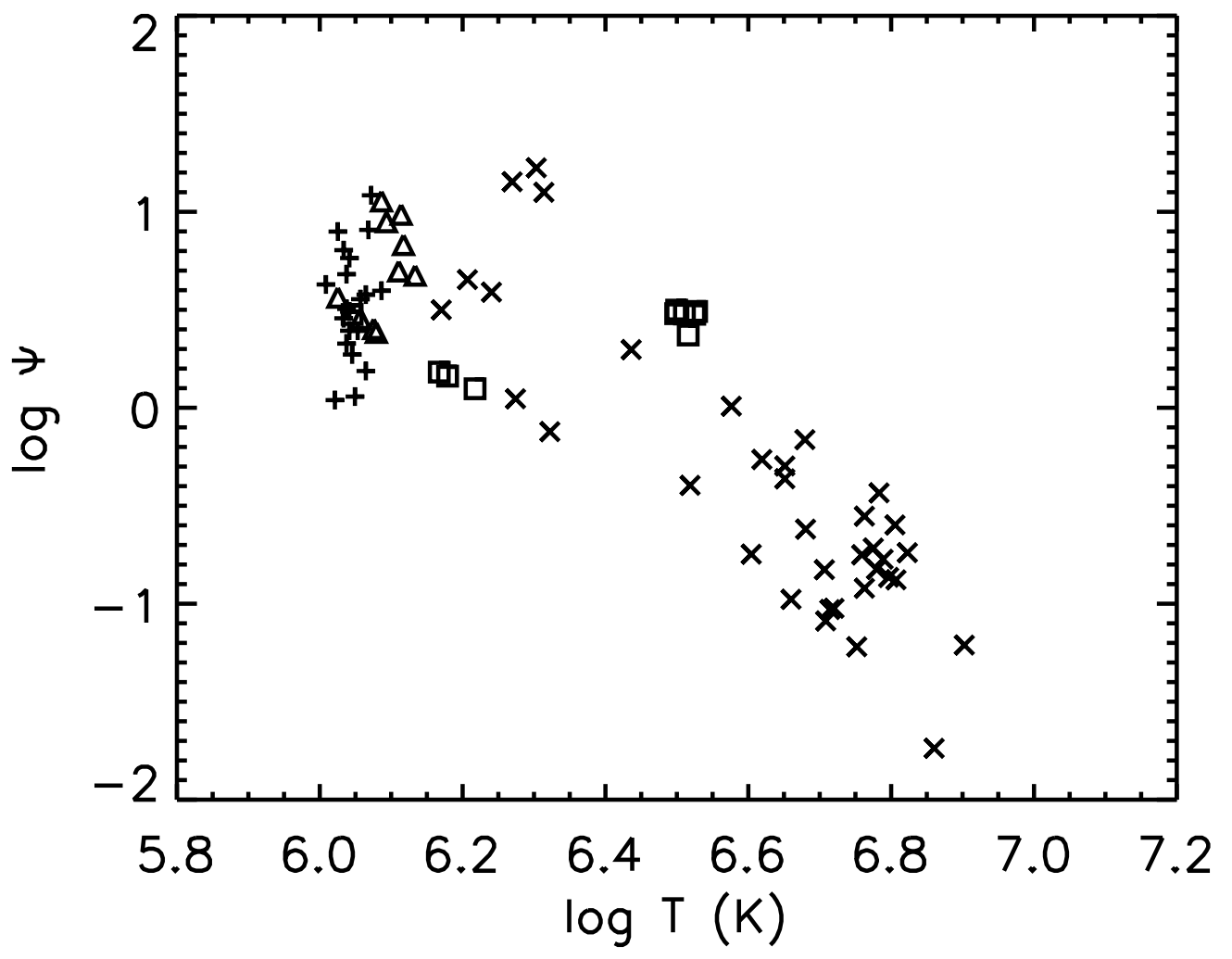

Fig. 7.- Excess density factor versus temperature for real loops observed by TRACE (pluses) and SXT (crosses) and for model loops with simulated observations by TRACE (diamonds) and SXT (squares). 


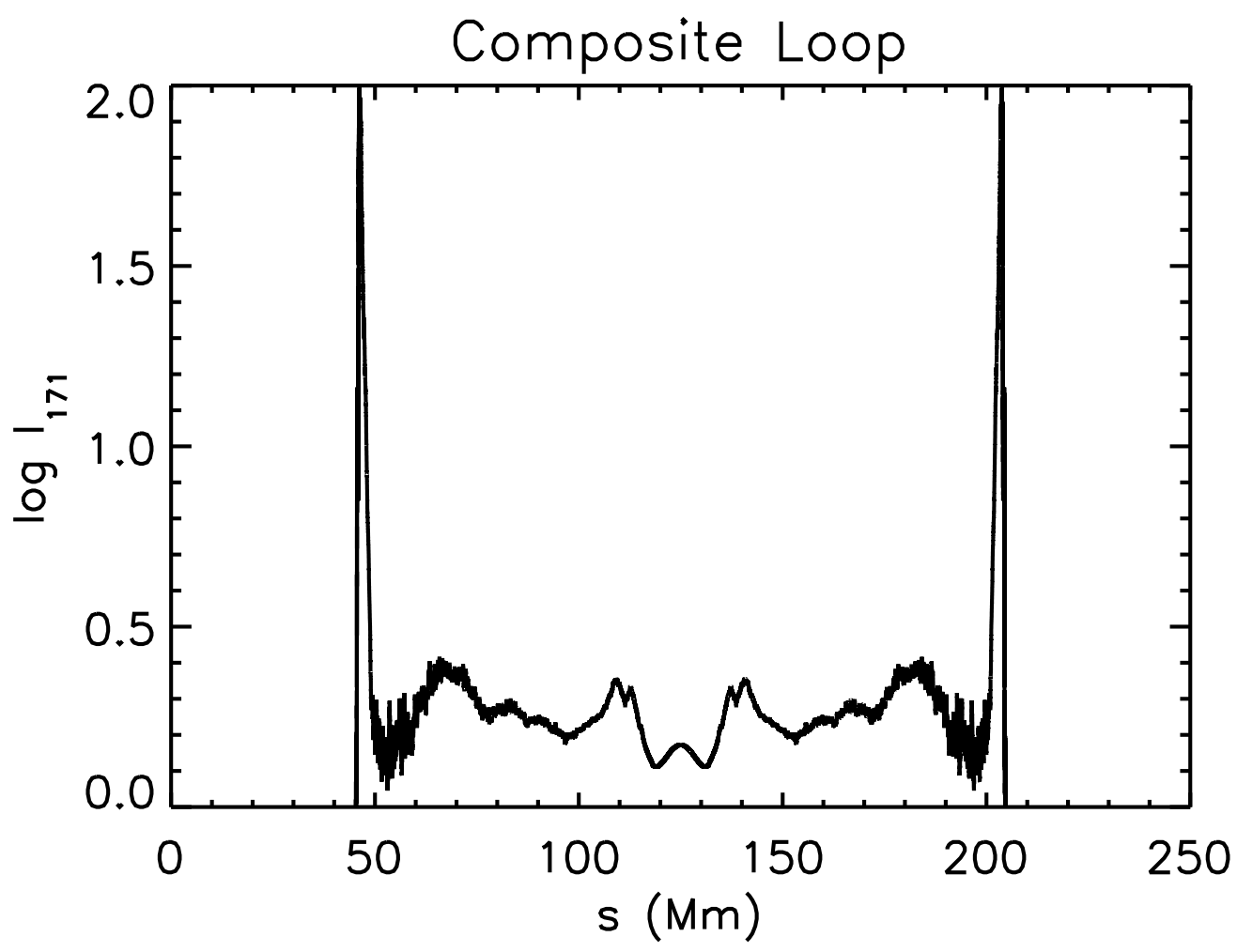

Fig. 8.- Logarithm of the TRACE 171 intensity versus position for the composite loop with strong heating. 


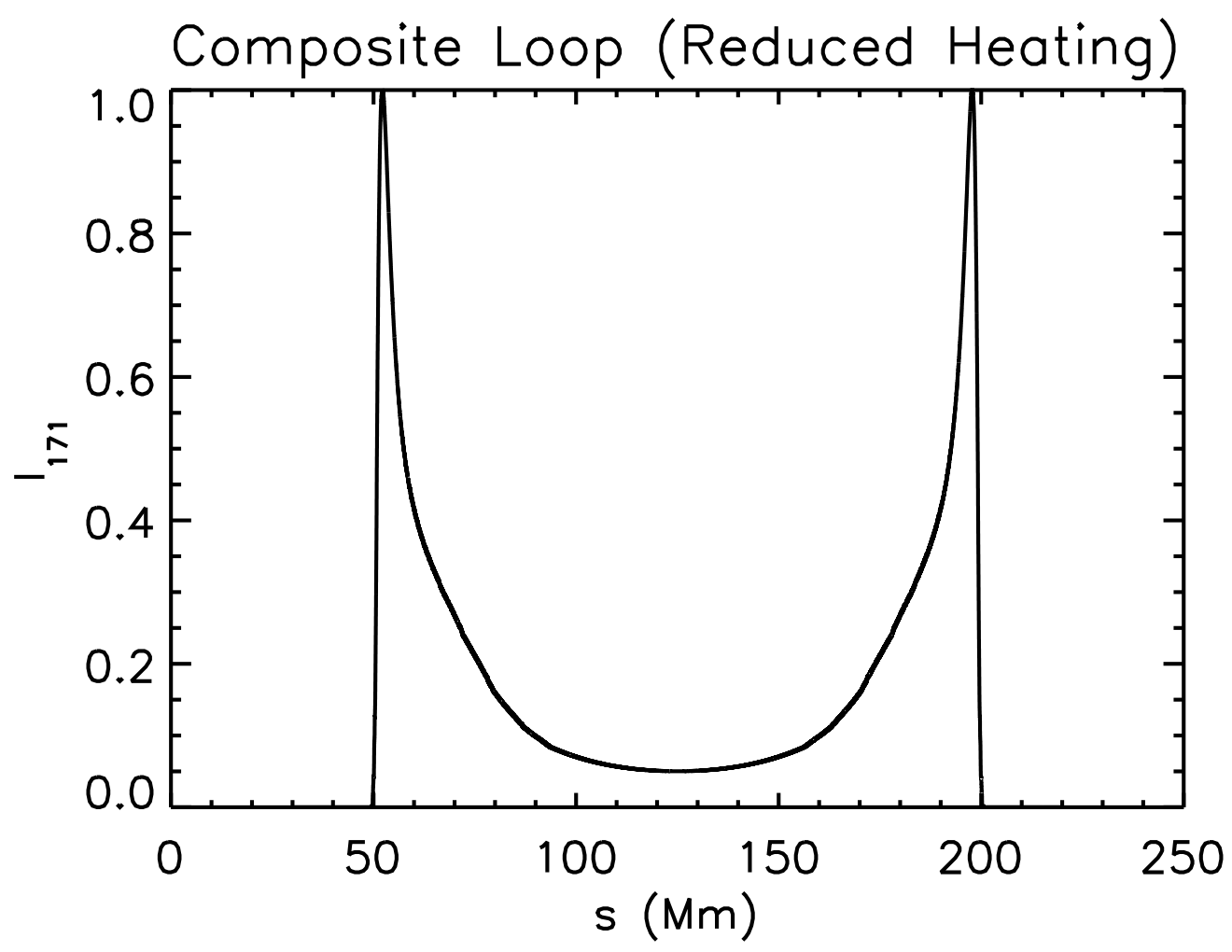

Fig. 9.- TRACE 171 intensity versus position for the composite loop with weak heating. 


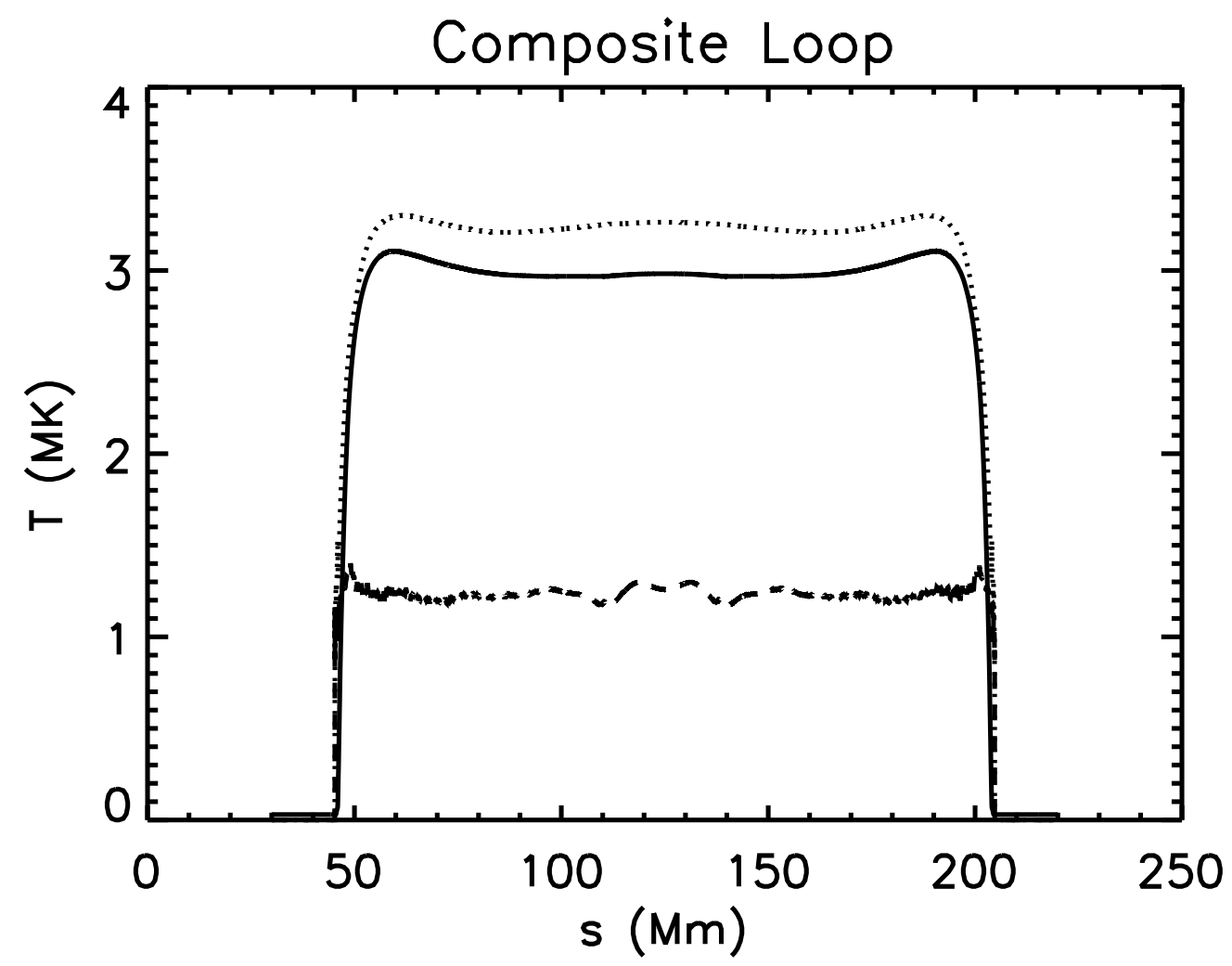

Fig. 10.- Temperature versus position for the composite loop with strong heating: average of the actual temperatures (solid), simulated TRACE temperature (dashed), and simulated SXT temperature (dotted). 